\title{
Macelignan Inhibits Melanosome Transfer Mediated by Protease- Activated Receptor-2 in Keratinocytes
}

\author{
Eun-Jung ChoI, ${ }^{a}$ Young-Gyu KAnG, ${ }^{b}$ Jaekyung KIM, ${ }^{a}$ and Jae-Kwan HwanG ${ }^{*}, a, b$ \\ ${ }^{a}$ Department of Biotechnology, College of Life Science and Biotechnology, Yonsei University; and ${ }^{b}$ Department of \\ Biomaterials Science and Engineering, Yonsei University; Seoul 120-749, South Korea. \\ Received November 11, 2010; accepted January 18, 2011; published online February 18,2011
}

\begin{abstract}
Skin pigmentation is the result of melanosome transfer from melanocytes to keratinocytes. Protease-activated receptor-2 (PAR-2) is a key mediator of melanosome transfer, which occurs as the melanocyte extends its dendrite toward surrounding keratinocytes that take up melanosomes by phagocytosis. We investigated the effects of macelignan isolated from Myristica fragrans HoutT. (nutmeg) on melanosome transfer and the regulation of PAR-2 in human keratinocytes (HaCaT). HaCaT cells stimulated by the PAR-2-activating peptide Ser-Leu-Ile-

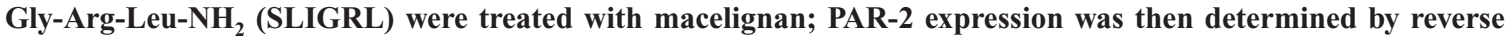
transcription-polymerase chain reaction (RT-PCR), Western blot, and immunocytochemistry. We evaluated the effects of macelignan on calcium mobilization and keratinocyte phagocytosis. In addition, B16F10 melanoma cells and keratinocytes were co-cultured to assess the effects of macelignan on prostaglandin $\mathrm{E}_{2}\left(\mathrm{PGE}_{2}\right)$ secretion and subsequent dendrite formation. Macelignan decreased HaCaT PAR-2 mRNA and protein levels in a dose-dependent manner. Furthermore, macelignan markedly reduced intracellular calcium mobilization and significantly downregulated keratinocyte phagocytosis, as shown by decreased ingestion of Escherichia coli bioparticles and fluorescent microspheres. In co-culture experiments, macelignan reduced keratinocyte $\mathrm{PGE}_{2}$ secretion, thereby preventing dendrite formation in B16F10 melanoma cells compared with SLIGRL-treated controls. Macelignan inhibits melanosome transfer by downregulating PAR-2, thereby reducing keratinocyte phagocytosis and $\mathrm{PGE}_{2}$ secretion, which in turn inhibits dendrite formation in B16F10 melanoma cells. Taken together, our findings suggest that macelignan could be used as a natural depigmenting agent to ameliorate hyperpigmentation.
\end{abstract} lization

Key words macelignan; protease-activated receptor-2; phagocytosis; skin pigmentation; melanosome transfer; calcium mobi-

Melanocytes are located in the basal layer of the epidermis, which separates the epidermis from the dermis; each melanocyte is surrounded by approximately 36 keratinocytes. ${ }^{1)}$ Melanin is produced in melanocytes and stored inside an organelle called the melanosome. Melanized melanosomes are then transferred from melanocytes by dendrites to neighboring keratinocytes, where melanosomes are distributed for skin pigmentation. ${ }^{2)}$ Although the chemical basis of melanin production and melanin trafficking are well documented, the molecular mechanism of melanosome transfer is not yet understood. ${ }^{3)}$

Several mechanisms have been suggested for melanosome transfer from the tips of melanocyte dendrites to keratinocytes, including phagocytosis, direct injection, release of melanosomes into intracellular spaces followed by endocytosis, and keratinocyte-melanocyte membrane fusion. ${ }^{4)}$ Phagocytosis by keratinocytes is thought to be the most probable means of melanosome transfer, with protease-activated receptor-2 (PAR-2) as a key mediator of the process. ${ }^{5-7)}$

PAR-2 is a seven-transmembrane, G-protein-coupled receptor activated by cleavage of its extracellular domain by serine proteases (e.g., trypsin or mast cell tryptase) ${ }^{8,9)}$ Unlike the activation of thrombin receptors (PAR-1, PAR-3, and PAR-4), the cleavage of PAR-2 exposes a new N-terminus that acts as a tethered ligand. ${ }^{1)}$ PAR-2 is also activated by the synthetic peptide, Ser-Leu-Ile-Gly-Arg-Leu- $\mathrm{NH}_{2}$ (SLIGRL), which corresponds to the newly created $\mathrm{N}$-terminus of the cleaved receptor. ${ }^{4}$ PAR-2 is expressed on keratinocytes but not on melanocytes; modulation of PAR-2 activity enhances or decreases melanosome transfer, thereby regulating skin pigmentation. ${ }^{10)}$
Skin pigment disorders such as melasma and postinflammatory hyperpigmentation are characterized by increased melanosome production and accumulation. ${ }^{11)}$ Hyperpigmentation has psychosocial and cosmetic relevance; therefore, considerable effort has been devoted to screening effective depigmenting agents. The ideal depigmenting agent exerts rapid, potent, and selective bleaching effects on melanocytes, has no side-effects, and permanently removes undesired pigment. ${ }^{12)}$ Tyrosinase is the key enzyme in melanogenesis, initiating a cascade of reactions that convert tyrosine to melanin; accordingly, most available topical depigmenting agents target tyrosinase. ${ }^{13)}$ Most of these drugs temporarily lighten pigmentation, but the hyperpigmentation recurs when the drug is discontinued. Although advances have been made, safer and more effective depigmenting agents are needed. ${ }^{14)}$

Macelignan, a natural lignan compound isolated from Myristica fragrans HouTt. (nutmeg), exerts anti-oxidant, anti-inflammatory, anti-carcinogenic, anti-diabetic, and hepatoprotective effects. ${ }^{15-17)}$ In addition, macelignan has been reported to inhibit melanin biosynthesis in murine melan-a melanocytes. ${ }^{18)}$ However, the effects of macelignan on melanosome transfer have not been determined. The present study aimed to elucidate the ability of macelignan to block PAR-2-mediated melanosome transfer in human keratinocytes.

\section{MATERIALS AND METHODS}

Plant Material Dried seeds of Myristica fragrans HouTt. (nutmeg) were collected in Jakarta, Indonesia, and 


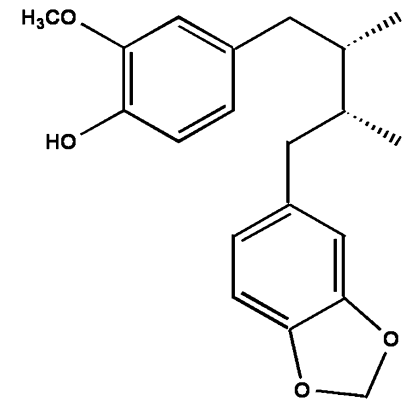

Fig. 1. The Chemical Structure of Macelignan

identified by Dr. Nam-In Baek, Department of Oriental Medicinal Materials and Processing, Kyunghee University (Yongin, Korea). A voucher specimen is deposited in the Department of Biotechnology, Yonsei University (Seoul, Korea).

Isolation of Macelignan The ground nutmeg (100 g) was extracted with $100 \%$ ethanol $(500 \mathrm{ml})$; the extract (18.3 g) was subjected to silica gel column chromatography (60, 70-230 mesh, Merck \& Co., Whitehouse Station, NJ, U.S.A.) and eluted with $n$-hexane and ethyl acetate solution $(5: 1, \mathrm{v} / \mathrm{v})$ to obtain four fractions (Fr. I-IV). Fr. III was eluted with $80 \%$ methanol with a Rp-18 column (LiChroprep $^{\circledR}, 25-40 \mu \mathrm{m}$, Merck \& Co.). Fr. III-B was finally obtained as a single compound $(2.7 \mathrm{~g})$. Data obtained from proton nuclear magnetic resonance $\left({ }^{1} \mathrm{H}-\mathrm{NMR}\right),{ }^{13} \mathrm{C}$ NMR, and fast atom bombardment mass spectrometry (FABMS) analysis of compound Fr. III-B were compared with published data, ${ }^{19}$ which suggested that the chemical structure was macelignan (Fig. 1).

Synthetic Peptide and Reagents All chemicals were obtained from Sigma-Aldrich Co. (St. Louis, MO, U.S.A.) unless otherwise specified. SLIGRL-NH $\mathrm{N}_{2}$, a PAR-2-activating peptide, was synthesized by COSMO Genetech (Seoul, Korea). Antibodies were obtained from Santa Cruz Biotechnology (Santa Cruz, CA, U.S.A.), and enhanced chemoluminescence (ECL) solution was purchased from Amersham Pharmacia Biotech (Piscataway, NJ, U.S.A.).

Cell Culture The spontaneously transformed human keratinocyte cell line HaCaT was kindly provided by Dr. Kwang Hoon Lee (Department of Dermatology and Cutaneous Biology Research Institute, Yonsei University College of Medicine, Seoul, Korea). The human keratinocyte cell line HaCaT (passages 7-15) was cultured in Dulbecco's modified Eagle's medium (DMEM; Gibco, Rockville, MD, U.S.A.) supplemented with $10 \%$ heat-inactivated fetal bovine serum (FBS; Gibco), $100 \mathrm{U} / \mathrm{ml}$ penicillin A, and $100 \mathrm{U} / \mathrm{ml}$ streptomycin. Cells were cultured at $37^{\circ} \mathrm{C}$ in a humidified atmosphere of $5 \% \mathrm{CO}_{2}$. HaCaT cells were grown to 40 to $60 \%$ confluence and then stimulated with SLIGRL $(10 \mu \mathrm{M})$ for $24 \mathrm{~h}$. Stimulated cells were treated with macelignan in $0.1 \%$ (v/v) dimethyl sulfoxide (DMSO) or DMSO alone (control) for $24 \mathrm{~h}$ in serum-free culture medium. B16F10 murine melanoma cells (passages 7-15) were obtained from American Type Culture Collection (ATCC, Manassas, VA, U.S.A.) and grown in the same medium and conditions. Both cell lines were maintained at less than $80 \%$ confluency.

Determination of Cell Viability Cell growth and viability were measured with the 3-[4,5-dimethylthiazol-2-yl]-2,5diphenyltetrazolium bromide (MTT) colorimetric assay
(Sigma-Aldrich). Briefly, HaCaT cells were seeded in a 96well microtiter plate $\left(1 \times 10^{4}\right.$ cells/well in $200 \mu \mathrm{l}$ DMEM) (Corning Costar, Cambridge, MA, U.S.A.) and incubated for $24 \mathrm{~h}$ to allow the cells to attach. To determine the effect of macelignan on $\mathrm{HaCaT}$ cell viability, cells were stimulated with SLIGRL $(10 \mu \mathrm{M})$ and macelignan for $24 \mathrm{~h}$. The culture medium was replaced with $100 \mu \mathrm{l}$ MTT $(1 \mathrm{mg} / \mathrm{ml})$ and incubated for $4 \mathrm{~h}$ at $37^{\circ} \mathrm{C}$. The MTT solution was removed by aspiration and the insoluble formazan dye was solubilized in $200 \mu \mathrm{l}$ DMSO. Spectrophotometric absorbance was measured at $550 \mathrm{~nm}$ with a tunable microplate reader (Versa Max, Sunnyvale, CA, U.S.A.).

Reverse Transcription-Polymerase Chain Reaction Total RNA was isolated from the cell pellet with Trizol reagent (Invitrogen, Carlsbad, CA, U.S.A.) and quantified by spectrophotometry at $260 \mathrm{~nm}$. The cDNA was synthesized with $1 \mu \mathrm{g}$ of total RNA, oligo (dT), and Reverse Transcription Premix (ELPIS-Biotech, Daejeon, Korea) in a $20 \mu \mathrm{l} \mathrm{re-}$ action. The primer sequences were: PAR-2 forward $5^{\prime}$-CCT GGC CAT GTA CCT GAT CT-3', PAR-2 reverse 5'-GAC ACT TCG GCA AAG GAG AG-3', $\beta$-actin forward 5'-ATC ATT GCT CCT CCT GAG CG-3', and $\beta$-actin reverse $5^{\prime}$ CAG CTC AGT AAC AGT CCG CC-3'. For PAR-2, polymerase chain reaction (PCR) amplification consisted of 30 cycles: denaturation at $95^{\circ} \mathrm{C}$ for $1 \mathrm{~min}$, annealing at $58^{\circ} \mathrm{C}$ for $1 \mathrm{~min}$, extension at $72^{\circ} \mathrm{C}$ for $1 \mathrm{~min}$, followed by a final 5 min extension at $72^{\circ} \mathrm{C}$. For $\beta$-actin, PCR amplification consisted of 25 cycles: denaturation at $95^{\circ} \mathrm{C}$ for $1 \mathrm{~min}$, annealing at $60^{\circ} \mathrm{C}$ for $1 \mathrm{~min}$, extension at $72^{\circ} \mathrm{C}$ for $1 \mathrm{~min}$, followed by a final $5 \mathrm{~min}$ extension at $72^{\circ} \mathrm{C}$. PCR products were resolved by $1.5 \%$ agarose gel electrophoresis and visualized with the LAS 3000 Bio Imaging Analysis System (Fuji Film, Tokyo, Japan). Relative band densities were determined with Multi Gauge Ver. 3.0 software (Fuji Film).

Western Blot Analysis $\mathrm{HaCaT}$ cells were lysed in radio immunoprecipitation assay (RIPA) buffer containing protease inhibitors and then held on ice for $10 \mathrm{~min}$. The lysate protein concentrations were determined with the Bradford protein assay (Bio-Rad Laboratories, Hercules, CA, U.S.A.). Equal amounts of protein $(60-100 \mu \mathrm{g})$ in each sample were loaded, separated by $10 \%$ sodium dodecyl sulfate-polyacrylamide gel electrophoresis (SDS-PAGE), and transferred to nitrocellulose membranes (Whatman $\mathrm{GmBH}$, Dassel, Germany). The membranes were blocked with 5\% skim milk in Tris-buffered saline containing $0.1 \%$ Tween-20 (TBST) for $1 \mathrm{~h}$ at room temperature and then incubated overnight with the PAR-2 primary antibody (dilution, $1: 500$ ) at $4{ }^{\circ} \mathrm{C}$. Bound antibodies were detected by horseradish peroxidase-conjugated secondary antibodies; signals were detected by enhanced chemoluminescence (ECL) and visualized with the LAS 3000 Bio Imaging Analysis System (Fuji Film). Relative band densities were determined by Multi Gauge Ver. 3.0 software (Fuji Film).

Calcium Mobilization Assay Cells were grown to confluence in black-wall clear-bottom 96-well plates (Corning Costar) for $24 \mathrm{~h}$. Before the assay, cells were incubated in serum-free medium containing macelignan for $6 \mathrm{~h}$. All assays were performed with the FLEXstation Calcium Plus assay kit (Molecular Devices) according to the manufacturer's instructions. Calcium assay solution was dissolved in Hanks' Balanced Salt Solution (HBSS; Invitrogen) containing $2.5 \mathrm{~mm}$ 
probenecid and $20 \mathrm{~mm} N$-(2-hydroxyethyl)piperazine- $N^{\prime}-2$ ethanesulfonic acid (HEPES). HaCaT cells were incubated with $100 \mu$ loading buffer for $1 \mathrm{~h}$ at $37^{\circ} \mathrm{C}$. After incubation, cells were stimulated with SLIGRL $(10 \mu \mathrm{m}$ in HBSS $)$ and intracellular calcium levels were determined at $25^{\circ} \mathrm{C}(485 \mathrm{~nm}$ excitation $/ 525 \mathrm{~nm}$ emission). Fluorescence intensity was recorded every $3 \mathrm{~s}$ for the first $3 \mathrm{~min}$ after SLIGRL addition. All experiments were repeated at least three times.

Quantitative Phagocytosis Assay Phagocytosis activity was quantified with the Vybrant Phagocytosis Assay (Molecular Probes, Eugene, OR, U.S.A.) according to the manufacturer's instructions. Briefly, HaCaT cells were seeded in a 96well microtiter plate $\left(5 \times 10^{3} /\right.$ well; Corning Costar). Cells were treated with SLIGRL $(10 \mu \mathrm{M})$ and macelignan $(10 \mu \mathrm{M})$ for $24 \mathrm{~h}$. After treatment, cells were incubated for $4 \mathrm{~h}$ with $100 \mu \mathrm{l}$ fluorescein-labeled Escherichia coli (K-12 strain) bioparticles. Then, the E. coli bioparticle suspension was aspirated, and extracellular fluorescence was quenched with $100 \mu 1$ Trypan Blue for 1 min. Following Trypan Blue aspiration, fluorescence was measured $(485 \mathrm{~nm}$ excitation $/ 538 \mathrm{~nm}$ emission) with a SpectraMax ${ }^{\circledR}$ Gemini microtiter plate reader (Molecular Device, Sunnyvale, CA, U.S.A.). Data was collected with SoftMax ${ }^{\circledR}$ Pro 5.3 software (Molecular Devices). Each experiment was performed with six replicate samples and repeated at least three times.

Microsphere-Based Phagocytosis Assay $\mathrm{HaCaT}$ cells were seeded onto coverslips in 24-well plates (Corning Costar), incubated overnight at $37^{\circ} \mathrm{C}$, and then treated with SLIGRL $(10 \mu \mathrm{M})$ and macelignan $(10 \mu \mathrm{M})$ for $24 \mathrm{~h}$. Cells were then serum-starved for $6 \mathrm{~h}$. FluoSpheres ${ }^{\circledR}$ carboxylatemodified red fluorescent microspheres $(1 \mu \mathrm{m}$ diameter; Invitrogen) were prepared $24 \mathrm{~h}$ before use according to the manufacturer's instructions. Cells were incubated with microspheres $(100 /$ cell $)$ at $37^{\circ} \mathrm{C}$ for $16 \mathrm{~h}$. The microspheres were then aspirated, and cells were incubated with $1 \mathrm{ml}$ FBS at $37^{\circ} \mathrm{C}$ for $15 \mathrm{~min}$. After extensive washing, nuclei were labeled with 4'6-diamino-2-phenylindole dihydrochloride (DAPI; Sigma-Aldrich) and images of the ingested beads were taken. Each experiment was performed in duplicate and repeated at least three times.

Immunocytochemistry and Confocal Microscopy All cell lines, cultured alone or in co-culture, were incubated overnight at $37^{\circ} \mathrm{C}$ and then serum-starved for $16 \mathrm{~h}$. After $24 \mathrm{~h}$ macelignan treatment $(10 \mu \mathrm{M})$, cells were fixed with $4 \%$ paraformaldehyde in phosphate buffered saline (PBS) for $10 \mathrm{~min}$ at room temperature and permeabilized with $0.2 \%$ Triton X-100 in PBS for 15 min. Cells were incubated with the following primary antibodies: anti-PAR-2 monoclonal antibody (1:50 in PBS; Santa Cruz Biotechnology) and antityrosinase polyclonal antibody (1:200 in PBS; Santa Cruz Biotechnology). After washing with PBS, proteins were visualized with the following secondary antibodies: goat antimouse immunoglobulin $\mathrm{G}$ (IgG)-rhodamine $(1: 200$ in PBS; Santa Cruz Biotechnology) and rabbit anti-goat IgG-fluorescein isothiocyanate (FITC) $(1: 300$ in PBS; Cell Signaling Technology, Beverly, MA, U.S.A.). To block non-specific binding, samples were incubated with $10 \%$ normal goat serum for $30 \mathrm{~min}$. After labeling nuclei with DAPI $(1: 5000$ in PBS), fluorescence was determined by confocal laser scanning microscopy (LSM 510, Carl Zeiss, Jena, Germany) and LSM 5 Image software (Carl Zeiss).
Co-culture Experiment $\mathrm{HaCaT}$ cells were seeded in the permeable inserts $\left(5 \times 10^{4}\right.$ cells/insert $)$ of 6 -well transwell plates $(0.4 \mu \mathrm{m}$ pore size polyester membrane coated with poly-D-lysine; Corning Costar, Bodenheim, Germany). B16F10 melanoma cells were seeded in the lower compartment of the transwell $\left(5 \times 10^{3}\right.$ cells/well). The two cell lines were cultured in the same well and shared the same medium without direct cell-to-cell contact, $\mathrm{HaCaT}$ cells in inserts placed above the B16F10 melanoma cells. The cells were incubated with SLIGRL $(10 \mu \mathrm{M})$ and macelignan $(10 \mu \mathrm{M})$ for $24 \mathrm{~h}$. The cell culture medium was analyzed for prostaglandin $\mathrm{E}_{2}\left(\mathrm{PGE}_{2}\right)$ secretion, and B16F10 melanoma cells in the lower compartment were fixed for immunocytochemistry.

Measurement of Prostaglandin $\mathbf{E}_{\mathbf{2}}\left(\mathbf{P G E}_{\mathbf{2}}\right)$ The $\mathrm{PGE}_{2}$ released by the $\mathrm{HaCaT}$ cells in co-culture was measured in the culture medium of the lower compartment of the transwell. The amount of $\mathrm{PGE}_{2}$ released was determined with an enzyme-linked immunosorbent assay (ELISA) kit (R\&D Systems, Minneapolis, MI, U.S.A.), according to the manufacturer's instructions.

Dendricity Assay Dendricity assay was quantitatively evaluated according to the method described previously. ${ }^{20,21)}$ Following treatment with SLIGRL and macelignan, B16F10 melanoma cells in the lower compartment of the transwell were fixed with $4 \%$ paraformaldehyde in PBS. The number of dendrites per cells was determined by microscopy ( $40 \times$ magnification). For each cell in the field of view, the length of the dendrite was measured from the cell nucleus to the dendrite tip using LSM 5 Image software (Carl Zeiss); approximately 60 cells were analyzed.

Statistical Analysis All data were expressed as the mean \pm standard deviation (S.D.). The evaluation of statistical significance was performed by one-way analysis of variance (ANOVA). The difference between treated groups and control groups were analyzed by Scheffe test (SPSS 12.0, SPSS Inc., Chicago, IL, U.S.A.). ${ }^{*} p<0.01, * p<0.05, * * p<0.01$ were considered statistically significant.

\section{RESULTS}

Macelignan Cytotoxicity in HaCaT Cells The effect of macelignan on $\mathrm{HaCaT}$ cell viability was determined by MTT colorimetric assay. Cells were stimulated with the synthetic PAR-2-activating peptide SLIGRL $(10 \mu \mathrm{M})$ and treated with macelignan $(1-50 \mu \mathrm{M})$ for $24 \mathrm{~h}$ (Fig. 2). Cell viability was not affected by macelignan concentrations lower than $10 \mu \mathrm{M}$. For that reason, we used $1-10 \mu \mathrm{m}$ macelignan for subsequent assays.

The Effects of Macelignan on PAR-2 Expression SLIGRL significantly increased the level of PAR-2 mRNA, which was reduced by macelignan $(1-10 \mu \mathrm{M})$ in a dose-dependent manner (Fig. 3A) SLIGRL-induced PAR-2 protein expression was also reduced to basal levels by macelignan (Fig. 3B). Immunocytochemistry analysis showed that SLIGRL-induced PAR-2 expression was markedly reduced by $10 \mu \mathrm{m}$ macelignan (Fig. 3C). These results indicate that macelignan inhibits SLIGRL-induced PAR-2 protein and mRNA expression.

The Effects of Macelignan on PAR-2 Mediated Phagocytosis In human keratinocytes, PAR-2 activation results in intracellular calcium mobilization. ${ }^{22)}$ We observed an imme- 
diate increase in fluorescence after SLIGRL treatment, indicating calcium mobilization; however, macelignan pretreatment dose-dependently attenuated the SLIGRL-induced calcium influx (Fig. 4A). To assess the effect of macelignan on PAR-2-mediated keratinocyte phagocytosis, HaCaT cells

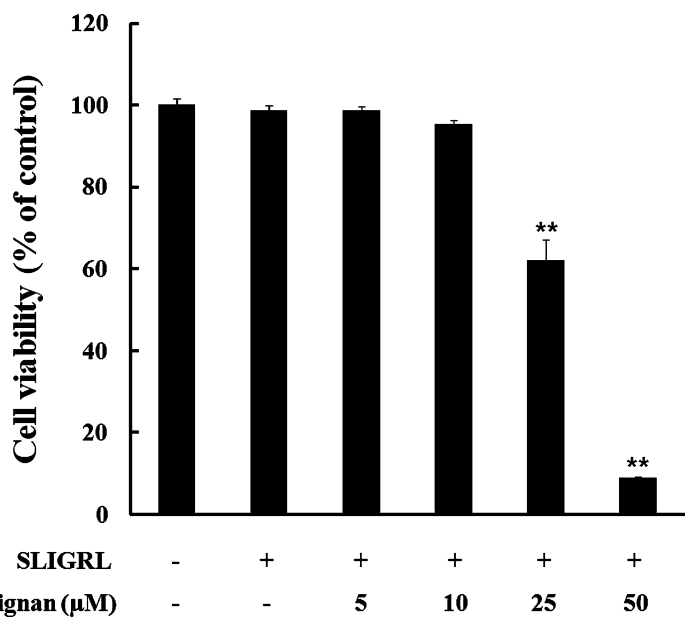

Fig. 2. Macelignan Cytotoxicity in HaCaT Cells Stimulated with the PAR2 Activating Peptide, SLIGRL (10 $\mu \mathrm{M})$

Data are expressed as \% control and each column represents the mean \pm S.D. of independent three tests. Asterisks indicate a significant difference compared with contro group, $* * p<0.01$ were stimulated with SLIGRL $(10 \mu \mathrm{M})$ and then incubated with fluorescently labeled $E$. coli bioparticles. SLIGRL increased the phagocytic response (Fig. 4B), but this effect was significantly attenuated by macelignan. To further elucidate the effects of macelignan on PAR-2-mediated phagocytosis, $\mathrm{HaCaT}$ cells were treated with SLIGRL $(10 \mu \mathrm{M})$ alone or with macelignan $(10 \mu \mathrm{M})$, and then incubated with fluorescently labeled microspheres $(1 \mu \mathrm{m}$ diameter $)$. Consistent with its effect on phagocytosis of $E$. coli bioparticles, macelignan treatment reduced the number of microspheres in the keratinocyte perinuclear area and cytoplasm compared with the control (Fig. 4C). These results indicate that macelignan inhibits keratinocyte phagocytosis.

The Effects of Macelignan on Dendrite Formation PAR-2 activation in keratinocytes stimulates $\mathrm{PGE}_{2}$ release, which acts as paracrine factor to stimulate melanocyte dendrite formation. ${ }^{23,24)}$ In co-culture, $10 \mu \mathrm{M}$ macelignan strongly inhibited $\mathrm{PGE}_{2}$ secretion from HaCaT cells (Fig. 5A). SLIGRL stimulation increased $\mathrm{PGE}_{2}$ secretion (2942.92 $\mathrm{pg} / \mathrm{ml} \pm 33.9)$ compared with the control $(1173.38 \mathrm{pg} / \mathrm{ml} \pm$ 220.9); however, macelignan significantly reduced SLIGRLinduced $\mathrm{PGE}_{2}$ secretion to $1840.27 \mathrm{pg} / \mathrm{ml} \pm 145.2$. Dendrite formation includes an increase in dendrite length as well as the number of dendrites. Macelignan significantly decreased the percentage of $\mathrm{B} 16 \mathrm{~F} 10$ melanoma cells with more than two dendrites in the co-culture system (Fig. 5B). Almost $80 \%$ of SLIGRL-treated cells had more than two dendrites,
(A)

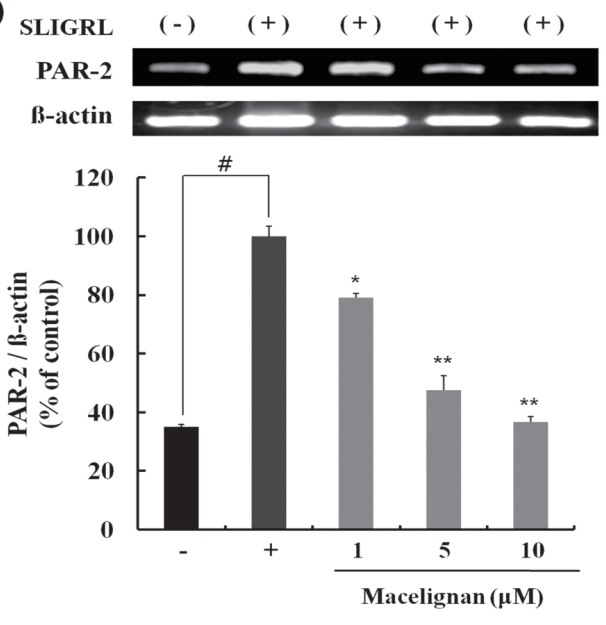

(B)

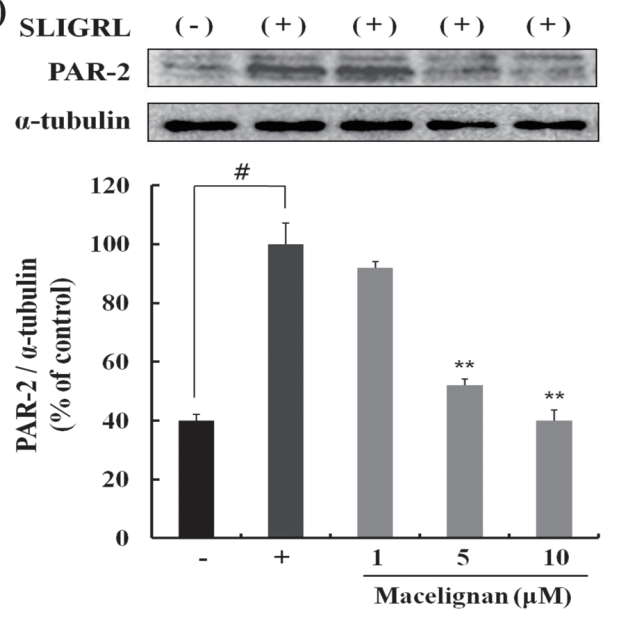

(C)

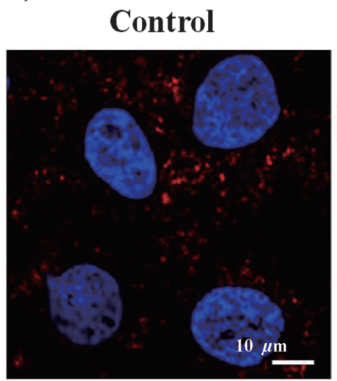

SLIGRL

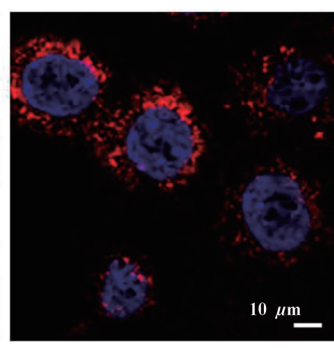

SLIGRL+

Macelignan

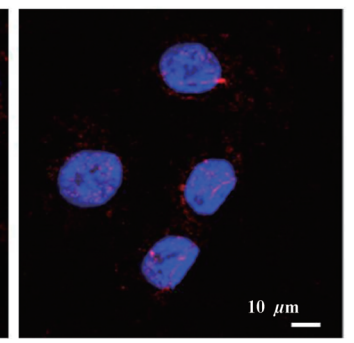

Fig. 3. The Effects of Macelignan on PAR-2 Expression

HaCaT cells were stimulated with the SLIGRL $(10 \mu \mathrm{M})$, and treated with macelignan. (A) PAR-2 mRNA levels were determined by reverse transcription-polymerase chain reaction. (B) Western blot analysis of PAR-2 expression. (C) Immunocytochemistry analysis of PAR-2 expression in keratinocytes (rhodamine-PAR-2 [red]; DAPI, nucleus [blue]). Scale bar: $10 \mu \mathrm{m}$. Data are expressed as \% control and each column represents the mean \pm S.D. of independent three tests. Asterisks indicate a significant difference compared with control group, $\# p<0.01, * p<0.05, * * p<0.01$. 
(A)

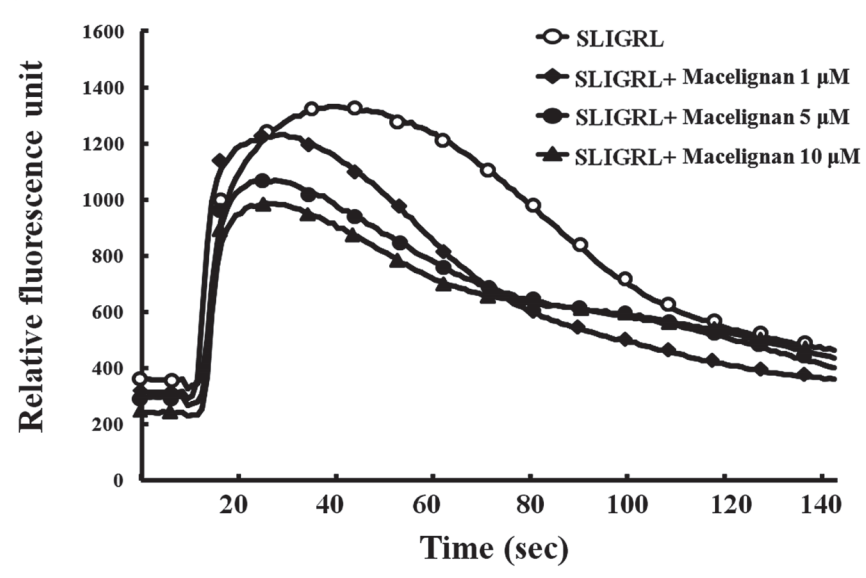

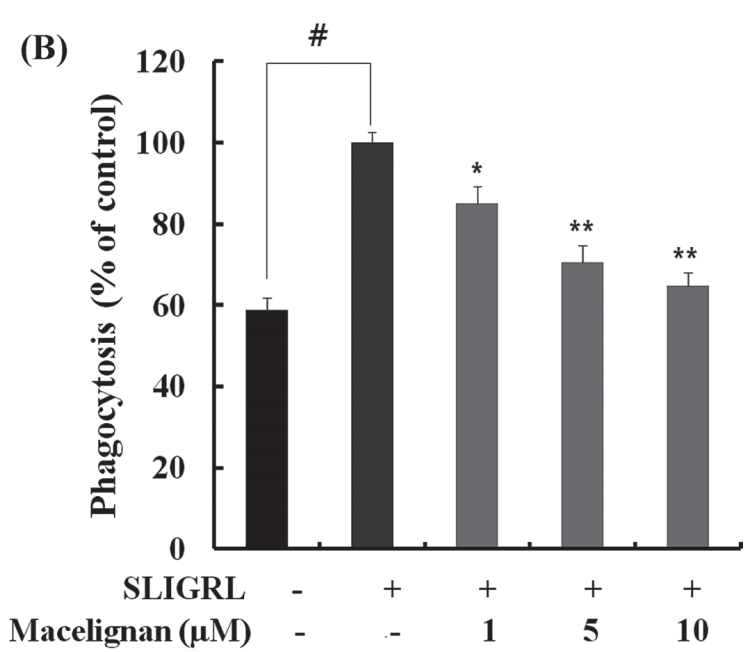

(C)

Control

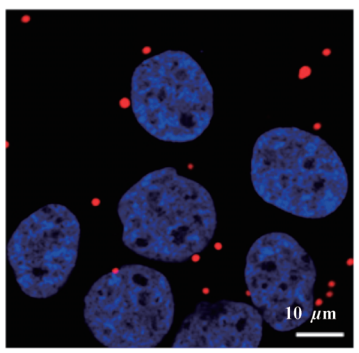

SLIGRL

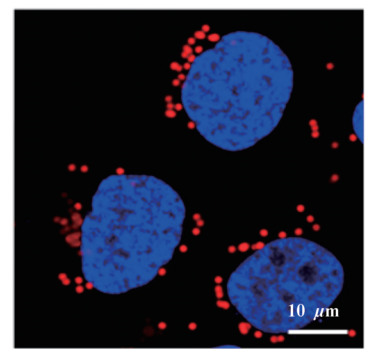

SLIGRL+ Macelignan

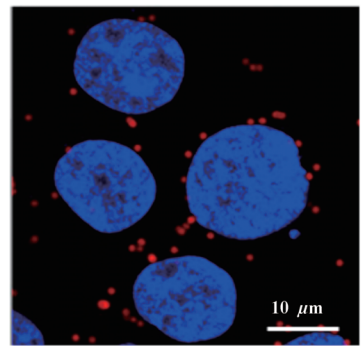

Fig. 4. The Effects of Macelignan on PAR-2 Mediated Phagocytosis

(A) PAR-2 activation in keratinocytes as assessed by calcium mobilization assay. (B) Quantitative phagocytosis assay with labeled E. coli bioparticles. Each experiment was performed with six replicates and repeated at least three times. (C) Immunocytochemical analysis of phagocytosis with fluorescently labeled microspheres (1 $\mu \mathrm{m}$ microspheres [red]; DAPI, nucleus [blue]). Data are expressed as \% control and each column represents the mean \pm S.D. of independent three tests. Asterisks indicate a significant difference compared with control group, $\# p<0.01, * p<0.05, * * p<0.01$.

whereas only $41 \%$ of cells treated with $10 \mu \mathrm{M}$ macelignan had more than two dendrites. Changes in dendrite length were also observed in B16F10 melanoma cells co-cultured with HaCaT cells. The mean dendrite length in SLIGRLstimulated cells was markedly increased compared with unstimulated control cells $(45 \pm 12 \mu \mathrm{m}$ vs. $18 \pm 6 \mu \mathrm{m})$, whereas the mean dendrite length in macelignan-treated cells was $21 \pm 9 \mu \mathrm{m}$ (Fig. 5C). PGE $_{2}$ secretion by SLIGRL-treated $\mathrm{HaCaT}$ keratinocytes increased the cytoplasmic tyrosinasepositive dots in B16F10 melanoma cells, indicating active dendrite formation (multiple long dendrites), whereas B16F10 melanoma cells co-cultured with macelignan-treated keratinocytes showed a typical bipolar morphology with few dendrites and short dendrite length (Fig. 5D). These results demonstrate that macelignan significantly reduced both the number and length of dendrites formed in B16F10 melanoma cells.

\section{DISCUSSION}

The phagocytic receptor PAR-2 is expressed in human keratinocytes and activated by UV irradiation. ${ }^{5,9)}$ In human keratinocytes, UV irradiation induces PAR-2 activation and expression and increase serine proteases secretion from the ke- ratinocytes, thereby further increasing the number of activated receptors. $^{1,4)}$ PAR-2 upregulates keratinocyte phagocytosis and also induces release of inflammatory mediators such as prostaglandins (PGs) that affect melanocyte dendrite formation. ${ }^{23)}$ Because melanocytes are a minority cell population within the epidermis (each melanocyte is surrounded by approximately 36 keratinocytes), dendrite formation is essential for melanosome transfer. ${ }^{1,4,25)}$ In the present study, we determined the effects of macelignan on PAR-2 mediated keratinocyte phagocytosis and dendrite formation in B16F10 melanoma cells.

Macelignan reduced the SLIGRL increased PAR-2 mRNA and protein levels almost to basal levels. High PAR-2 expression indicates increased keratinocyte phagocytosis, ${ }^{4,6}$ thus macelignan reduces melanosome transfer by downregulating PAR-2 in keratinocytes.

Few studies have described the cellular signaling pathways that regulate PAR-2; however, the earliest known event after PAR-2 activation is calcium release from the endoplasmic reticulum. ${ }^{24)}$ The elevated intracellular calcium levels induce cytoskeletal protein reorganization and cell surface morphology changes, which are important in keratinocyte phagocytosis. ${ }^{6,7)}$ Macelignan dose-dependently inhibited calcium mobilization in SLIGRL-treated HaCaT cells, suggesting that 
(A)

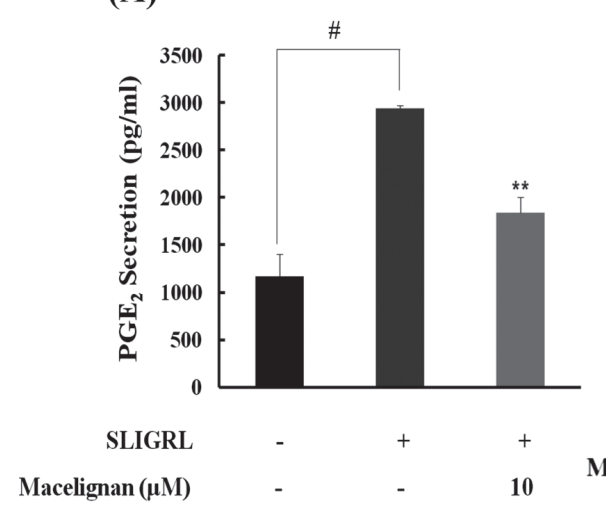

(B)

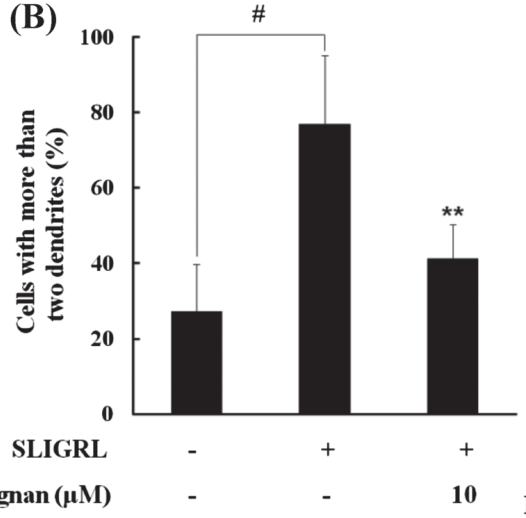

(C)

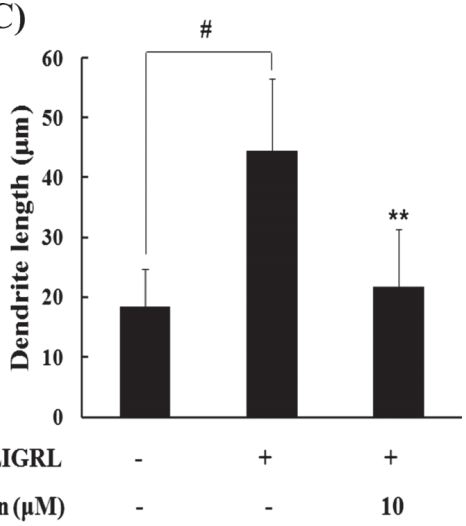

(D)

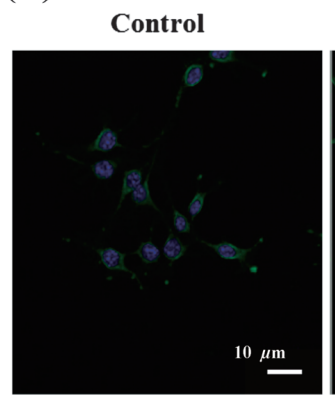

SLIGRL

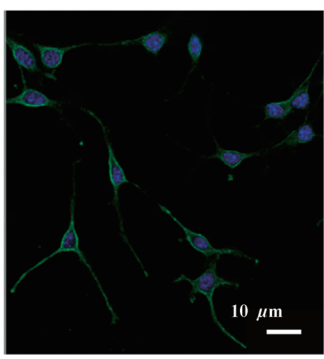

SLIGRL +

Macelignan

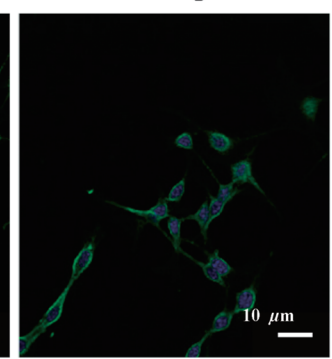

Fig. 5. The Effects of Macelignan on Dendrite Formation

(A) $\mathrm{PGE}_{2}$ secretion from SLIGRL stimulated HaCaT cells. $\mathrm{PGE}_{2}$ secretion into the cell culture medium was determined by enzyme-linked immunosorbent assay. Macelignan decreased (B) the percentage of cells with more than two dendrites and (C) dendrite length. (D) Immunocytochemical analysis of B16F10 melanoma cells dendrite formation (FITCtyrosinase [green]; DAPI, nucleus [blue]). Scale bar: $10 \mu \mathrm{m}$. Studies were performed in duplicate and repeated at least three times. Data are expressed as mean \pm S.D. of independent three tests. Asterisks indicate a significant difference compared with control group, $\# p<0.01, * * p<0.01$.

macelignan attenuates keratinocyte phagocytosis. Indeed, a significant decrease in $E$. coli bioparticle phagocytosis was observed after macelignan treatment. Similarly, macelignan reduced SLIGRL-induced microsphere ingestion. Because the amount of melanosome transfer contributes to skin pigmentation, ${ }^{26}$ ) these observations strongly suggest that macelignan may reduce hyperpigmentation through PAR-2 inhibition.

As previously mentioned, activated PAR-2 in keratinocytes stimulates secretion of $\mathrm{PGs},{ }^{23,25)}$ which are lipid hormones synthesized by the cyclooxygenase- $2(\mathrm{COX}-2)$ pathway from arachidonic acid. ${ }^{27)} \mathrm{PGE}_{2}$, the primary prostaglandin released after PAR-2 activation, is a potent stimulus for melanocyte dendrite formation. ${ }^{19,25,27)}$ In the present study, macelignan significantly reduced $\mathrm{PGE}_{2}$ secretion by keratinocytes co-cultured with B16F10 melanoma cells. This finding is consistent with that of our previous study, which demonstrated that macelignan decreased COX-2 expression in UVB-irradiated $\mathrm{HaCaT}$ cells through mitogen-activated protein kinase (MAPK) signaling and the phosphoinositide 3-kinase (PI3K)/Akt pathway. ${ }^{15)}$ Thus lower $\mathrm{PGE}_{2}$ secretion may be the mechanism by which macelignan inhibits dendrite formation in B16F10 melanoma cells.

Alternatively, macelignan may regulate dendrite formation by altering calcium mobilization. PAR-2 induces COX-2 expression through the $\mathrm{IP}_{3} /$ calcium pathway as well as the MAPK or PI3K/Akt pathways. ${ }^{28,29)}$ Macelignan may decrease $\mathrm{PGE}_{2}$ secretion by reducing calcium influx. Therefore, the inhibitory effects of macelignan on dendrite formation in B16F10 melanoma cells may be associated with suppression of prostaglandin synthesis and calcium influx in keratinocytes.

Several studies have described melanosome transfer inhibitors from natural sources including soybean extract, centaureidin, and niacinamide. Soybean extract contains small serine protease inhibitors, such as soybean trypsin inhibitor (STI), which inhibit PAR-2 activation by blocking cleavage of its extracellular domain. ${ }^{6}$ In keratinocyte-melanocyte cocultures, STI decreased tyrosinase-related protein (TRP)-1 expression, but had no effect on tyrosinase expression. ${ }^{30}$ Centaureidin is a flavonoid isolated from Achillea millefolium that appears to induce melanocyte dendrite retraction by activating Rho, thus reducing melanosome transfer; however, centaureidin did not inhibit melanin synthesis. ${ }^{21)}$ The vitamin $\mathrm{B}_{3}$ derivative niacinamide blocks melanogenesis by inhibiting melanosome transfer through an unknown molecular mechanism; however, niacinamide did not alter tyrosinase activity in melanocytes. ${ }^{31)}$

We previously reported that macelignan exhibited antimelanogenic effects by downregulating the melanogenesisrelated factors tyrosinase, TRP-1, and TRP-2. ${ }^{18)}$ In the present study, macelignan also inhibited melanosome transfer by reducing keratinocyte phagocytosis and B16F10 melanoma cells dendrite formation. Taken together, our results suggest that macelignan reduces pigmentation not only by inhibiting melanin formation in melanocytes, but also by attenuating 
melanosome transfer from melanocytes to keratinocytes. Although additional studies are necessary to further characterize the mechanism underlying macelignan modulation of PAR-2, our findings strongly suggest that macelignan may serve as a potent natural anti-melanogenic agent to ameliorate skin hyperpigmentation.

Acknowledgement This work was supported in part by the Yonsei Biomolecule Research Initiative of the Brain Korea 21 Project.

\section{REFERENCES}

1) Seiberg M., Pigment Cell Res., 14, 236-242 (2001).

2) Yamaguchi Y., Hearing V. J., Biofactors, 35, 193-219 (2009).

3) Scott G., Leopardi S., Printup S., Madden B. C., J. Cell Sci., 115 $1441-151$ (2002).

4) Babiarz M. L., Chen N., Seiberg M., Lin C. B., Pigment Cell Res., 17, $241-251$ (2004).

5) Scott G., Deng A., Rodriguez B. C., Seiberg M., Han R., Babiarz M. L., Grizzle W., Bell W., Pentland A., J. Invest. Dermatol., 117, 14121420 (2001).

6) Sharlow E. R., Paine C. S., Babiarz M. L., Eisinger M., Shapiro S., Seiberg M., J. Cell Sci., 113, 3093-3101 (2000).

7) Scott G., Leopardi S., Parker L., Babiarz L., Seiberg M., Han R., J. Invest. Dermatol., 121, 529-541 (2003).

8) Coelho A. M., Ossovskaya V., Bunnett N. W., Curr. Med. Chem., 1, 61-72 (2003)

9) Seiberg M., Paine C., Sharlow E., Andrade-Gordon P., Costanzo M., Eisinger M., Shapiro S. S., J. Invest. Dermatol., 115, 162-167 (2000).

10) Boissy R. E., Exp. Dermatol., 12, 5-12 (2003).

11) Virador V. M., Muller J., Wu X., Abdel-Malek Z. A., Yu Z. X., Ferrans V. J., Kobayashi N., Wakamatsu K., Ito S., Hammer J. A., Hearing V. J., FASEB J., 16, 105-107 (2002).

12) Briganti S., Camera E., Picardo M., Pigment Cell Res., 15, 101-110 (2003).
13) Ebanks J. P., Wickett R. R., Boissy R. E., Int. J. Mol. Sci., 10, 40664087 (2009).

14) Lin J. W., Chiang H. M., Lin Y. C., Wen K. C., J. Food Drug Anal., 16, $1-10$ (2008).

15) Anggakusuma, Yanti, Hwang J. K., J. Dermatol. Sci., 57, 114-122 (2010).

16) Han K. L., Choi J. S., Lee J. Y., Song J., Joe M. K., Jung M. H., Hwang J. K., Diabetes, 57, 737-745 (2008).

17) Sohn J. H., Han K. L., Choo J. H., Hwang J. K., Biofactors, 29, 1-10 (2007).

18) Cho Y. M., Kim K. H., Shim J. S., Hwang J. K., Biol. Pharm. Bull., 31, 986-989 (2008).

19) Chung J. Y., Choo J. H., Lee M. H., Hwang J. K., Phytomedicine, 13, 261-266 (2006).

20) Miyazaki K., Pigment Cell Res., 17, 407-412 (2004).

21) Ito Y., Kanamaru A., Tada A., Biochim. Biophys. Acta, 1760, 487494 (2006).

22) Dery O., Corvera C., Steinhoff M., Bunnett N. W., Am. J. Physiol., 247, 1429-1452 (1998).

23) Scott G., Leopardi S., Printup S., Malhi N., Seiberg M., Lapoint R., J. Invest. Dermatol., 122, 1214-1224 (2004).

24) Park H. Y., Kosmadaki M., Yaar M., Gilchrest B. A., Cell Mol. Life Sci., 66, 1493-1506 (2008).

25) Scott G., Fricke A., Fender A., McClelland L., Jacobs S., Exp. Cell Res., 313, 3840-3850 (2007).

26) Ando H., Niki Y., Yoshida M., Kim J. H., Yoon T. J., Ichihashi M., Pigment Cell Melanoma Res., 23, 129-133 (2009).

27) Grew M., Trefzer U., Ballhorn A., Gyufko K., Henninger H., Krutmann J., J. Invest. Dermatol., 101, 528-531 (1993).

28) Macfarlane S. R., Sloss C. M., Cameron P., Kanke T., Mckenzie R. C., Plevin R., Br. J. Pharmacol., 145, 35-44 (2005).

29) Lin C. B., Chen N., Scarpa R., Guan F., Babiarz-Magee L., Liebel F., Li W. H., Kizoulis M., Shapiro S., Seiberg M., Pigment Cell Melanoma Res., 21, 172-183 (2008).

30) Paine C., Sharlow E., Liebel F., Eisinger M., Shapiro S., Seiberg M., J. Invest. Dermatol., 116, 587-595 (2001).

31) Greatens A., Hakozaki T., Koshoffer A., Epstein H., Schwemberger S., Bobcock G., Bissett D., Takiwaki H., Arase S., Wickett R. R., Boissy R. E., Exp. Dermatol., 14, 498-508 (2005). 